\title{
BMJ Open Network meta-analysis of four kinds of traditional Chinese exercise therapy in the treatment of type 2 diabetes: protocol for a systematic review
}

\author{
Yuqi Jia (D) , ${ }^{1}$ Ying Yu (D) ,' Hailiang Huang ${ }^{1}$
}

To cite: Jia Y, Yu Y, Huang $\mathrm{H}$. Network meta-analysis of four kinds of traditional Chinese exercise therapy in the treatment of type 2 diabetes: protocol for a systematic review. BMJ Open 2021;11:e048259. doi:10.1136/ bmjopen-2020-048259

- Prepublication history for this paper is available online. To view these files, please visit the journal online (http://dx.doi. org/10.1136/bmjopen-2020048259).

Received 22 December 2020 Accepted 28 June 2021

D Check for updates

(C) Author(s) (or their employer(s)) 2021. Re-use permitted under CC BY-NC. No commercial re-use. See rights and permissions. Published by BMJ.

${ }^{1}$ College of Rehabilitation Medicine, Shandong University of Traditional Chinese Medicine, Jinan, China

${ }^{2}$ College of Traditional Chinese Medicine, Shandong University of Traditional Chinese Medicine, Jinan, China

Correspondence to

Dr Hailiang Huang;

huanghailiang@sdutcm.edu.cn

\section{ABSTRACT}

Introduction Traditional Chinese exercise therapy, as one of the commonly used exercise interventions for the treatment of type 2 diabetes patients in China, has been proven effective by many clinical practices, but there is still a lack of evidence-based research. This study aims to integrate clinical randomised controlled correlations via network meta-analysis evidence.

Methods and analysis The comprehensive search included Chinese and other language databases such as the MEDLINE (PubMed), Web of Science, Excerpt Medica Database (EMBASE), The Cochrane Library, China National Knowledge Infrastructure, Wanfang Data Knowledge Service Platform, China Scientific Journal Database (VIP), China Biomedical Literature Database (CBM). Clinical randomised controlled trials of four traditional Chinese exercise therapies in the treatment of type 2 diabetes, including Tai Chi, Ba Duan Jin, Yi Jin Jing and Wu Qin $X i$ were retrieved. The search time was conducted from the establishment of the database to 30 October 2020. Two researchers screened the documents that met the inclusion criteria, extracted data according to the preset table, and evaluated the methodological quality of the included studies according to the quality evaluation tools recommended by the Cochrane System Reviewer Manual V.5.1. The R language and ADDIS statistical software were used to conduct statistics and analysis of intervention measures.

PROSPERO registration number CRD42020214786

\section{INTRODUCTION}

Type 2 diabetes (type 2 diabetes mellitus, T2DM), also known as non-insulin-dependent diabetes, is the most common form of clinical diabetes and mainly manifests as persistent hyperglycaemia. Its aetiology and pathogenes is are not yet clear, but the disease is mainly related to insulin secretion defects related to inflammation and metabolic stress, including the involvement of genetic factors. ${ }^{1}$ The significant pathophysiological feature of type 2 diabetes is the decrease in insulin's ability to regulate glucose metabolism (insulin resistance) and the decrease (or relative decrease) in insulin secretion caused
Strengths and limitations of this study

- This study may provide the best possible exercise therapy options and reliable evidence-based medicine for the clinical treatment of type 2 diabetes.

- To a certain extent, may provide some new insights for the auxiliary treatment of type 2 diabetes by exercise therapy.

- This study also has some limitations, including publication bias, clinical heterogeneity and selection bias.

by defects in pancreatic islet B cell function. ${ }^{2}$ As the natural course of the disease develops, the dependence on exogenous blood sugar control increases, which can affect multiple tissues and organs and can cause a variety of secondary complications. ${ }^{3}$ The disease can affect the large blood vessels (cerebrovascular disease, cardiovascular disease), microvasculature (podiatry, eye disease, kidney disease) and nerves (nephropathy, eye disease), ${ }^{4}$ reducing the quality of life of patients and endangering patients' lives.

With the improvement of living standards and changes in the human living environment, the incidence of T2DM is increasing. ${ }^{5}$ At present, the main methods of blood sugar control in patients with T2DM involve oral hypoglycaemic drugs and exogenous insulin supplements. Traditional treatment is highly dependent on drugs, and oral drugs and exogenous insulin can only temporarily maintain blood sugar or temporarily improve insulin sensitivity and are not curative. ${ }^{3}$

According to the latest version of the domestic guidelines for the prevention and treatment of type 2 diabetes (2017 edition), lifestyle intervention is the basic treatment measure for T2DM and is required for the treatment of diabetes; exercise plays an important role in the comprehensive management of patients with T2DM. ${ }^{2}$ According to 
the guidelines of the American College of Sports Medicine and the American Diabetes Association, it is recommended that patients with diabetes perform aerobic exercise. Patients with type 2 diabetes should perform at least $150 \mathrm{~min}$ of moderate-intensity or higher-intensity exercise a week, and they should perform it at least 3 days a week. ${ }^{6}$ Long-term regular aerobic exercise can improve the body weight and blood sugar and blood lipid levels of patients with type 2 diabetes and has an important role in the rehabilitation of type 2 diabetes and the prevention of complications. ${ }^{7}$

Traditional Chinese exercise therapy conforms to the characteristics of low-intensity and long-term aerobic exercise proposed by modern research. It is guided by the holistic concept of Chinese medicine as the main theoretical guide. By mobilising the human body's own potential, it can achieve the purpose of healing and strengthening the body, preventing and curing diseases, and it is a non-drug therapy for T2DM. ${ }^{89}$ In recent years, a number of randomised controlled studies have shown that four traditional exercise therapies play an irreplaceable role in the prevention of T2DM at all levels. Studies have found that Tai Chi can reduce damage to pancreatic islet cells by downregulating the expression of inflammatory cytokines, improving the body's sensitivity to insulin, improving insulin resistance, delaying the occurrence of diabetes complications and improving the quality of life of patients with T2DM. ${ }^{10}$ The application of Baduanjin in high-risk populations for diabetes can delay the time of glucose metabolism disorders, exert good stabilising effects on blood sugar and glycosylated haemoglobin $(\mathrm{GHb})$ and reduce regulatory fluctuations. ${ }^{11}$ Wuqinxi exercise can significantly improve the blood rheology of patients with T2DM and improve blood circulation function. ${ }^{12}$ Yijinjing can effectively regulate liver and spleen function in patients with T2DM and can help improve blood sugar levels. ${ }^{13}$

At present, studies in this area mostly use randomised controlled trials (RCTs) to verify the clinical efficacy of a single traditional exercise therapy on T2DM, and there is no evidence-based evaluation that compares the clinical efficacy of four traditional exercise therapies for T2DM at the same time. Therefore, this study selected four traditional exercise therapies commonly used in clinical practice as the research objects and used the method of network meta-analysis (NMA) to integrate relevant clinical evidence. After summarising different interventions in the same body of evidence, a quantitative comprehensive statistical analysis was performed to compare the clinical efficacy of four different traditional Chinese exercise therapies in the treatment of T2DM. Our purpose is to provide a reference for clinical treatment of T2DM to choose more effective exercise intervention therapies.

\section{METHODS AND ANALYSIS}

Patient and public involvement

No patients involved.

\section{Eligibility criteria}

The design of the inclusion and exclusion criteria of this study was based on the five main principles of the Participant-Intervention-Comparator-Outcomes-Study (PICOS) design search principle.

\section{Inclusion criteria}

Types of studies

The included literature type was RCTs, and there were no restrictions regarding the type of language, whether blinding was used or the requirements for allocation concealment. Most of the clinical trial reports in this study were from mainland China. As long as the included studies were approved by the local institution, we included the study in the scope of the study and registered it in the international database.

\section{Type of participants}

The patients all had type 2 diabetes, regardless of age, sex and race. The diagnostic criteria used for diabetes should comply with the 'China Type 2 Diabetes Guidelines (2010 Edition)', ${ }^{14}$ 'China Type 2 Diabetes Prevention Guidelines (2013 Edition), 15 'China Type 2 Diabetes Prevention Guidelines (2017 Edition)',, 'Diagnostic Guidelines for Diabetes Diagnosis and Classification Standards Revised by WHO in 1997'16 and 'Diagnostic Standards for Diabetes Made by WHO in 1999’.

\section{Types of interventions and comparators}

The control group received conventional basic treatment (eg, hyperglycaemic treatment, health education, voluntary exercise) without exercise intervention. The treatment group was treated with one of the four traditional exercise therapies when the diagnostic criteria, curative effect evaluation criteria and basic treatment were the same.

\section{Types of outcomes}

The predetermined main outcomes indicators were as follows: (1) blood glucose, including fasting blood glucose or fasting plasma glucose (FBG or FPG), 2-hour postprandial blood glucose or postprandial glucose (2hPG or PPG), and glycosylated haemoglobin or haemoglobin A1c (GHb or HbA1c); (b2) blood lipids, including total cholesterol (TC) and triglycerides (TG)s, highdensity lipoprotein (HDL-C), and low-density lipoprotein (LDL-C).

Secondary outcomes indicators included: (1) body mass index (BMI); (2) haemorheology indicators (whole blood low shear viscosity, whole blood high shear viscosity, plasma viscosity, haematocrit, erythrocyte sedimentation rate, red blood cell aggregation index, Fibrinogen(Fib), serum intercellular adhesion molecular(sICAM), and P-selectin(Ps)); (3) quality of life evaluations, including the Quality of Life-Specific Scale (DSQL) or Short Form 36-item Health Survey Scale(SF- 36) evaluations. 


\section{Exclusion criteria}

Self-control studies, case reports, review literature, duplicate publication, summary of experiences, animal experiment reasearch, studies with incomplete data, studies with patients that have other diseases, studies with no clear diagnosis or efficacy evalution standard, studies combined with other therapy that are different from the control group.

\section{Search methods for identification of studies Information sources}

A computer was used to conduct a comprehensive search for four traditional RCTs for the treatment of type 2 diabetes. The search time was from the establishment of the database to 30 October 2020. Computer databases searched included PubMed, Web of Science, EMBASE, The Cochrane Library, China National Knowledge Infrastructure, Wanfang Data Knowledge Service Platform, VIP.com (VIP) and China Biomedical Literature Database (CBM). Chinese search terms included Tai Chi, Ba Duan Jin, Yi Jin Jing, Wu Qin Xi, traditional exercises, Health Qigong, type 2 diabetes and random, etc. English search terms included Tai Chi, Ba Duan Jin, Yi Jin Jing, Wu Qin Xi, Traditional Exercises, Health Qigong, Type 2 Diabetes and Random, etc.

In addition, relevant references were tracked in the literature, and the corresponding authors were contacted when a complete report could not be obtained or when documents included incomplete relevant data. The best effort was made to ensure the comprehensiveness of the preliminary search work so as not to lose valuable research data. Example PubMed search strategies are shown in table 1 , and the terms matched the medical topic titles. according to the search modes of different databases, keywords could be combined with free words for a comprehensive search.

\section{Study selection and data extraction}

According to the above-mentioned electronic database search strategy, two researchers searched Chinese and English electronic databases, used Endnote X7 software to search for repeated studies, integrated the literature search results of different databases, established an information database and downloaded the full texts. Then,

\section{Table 1 Search strategy used inPubMed database}

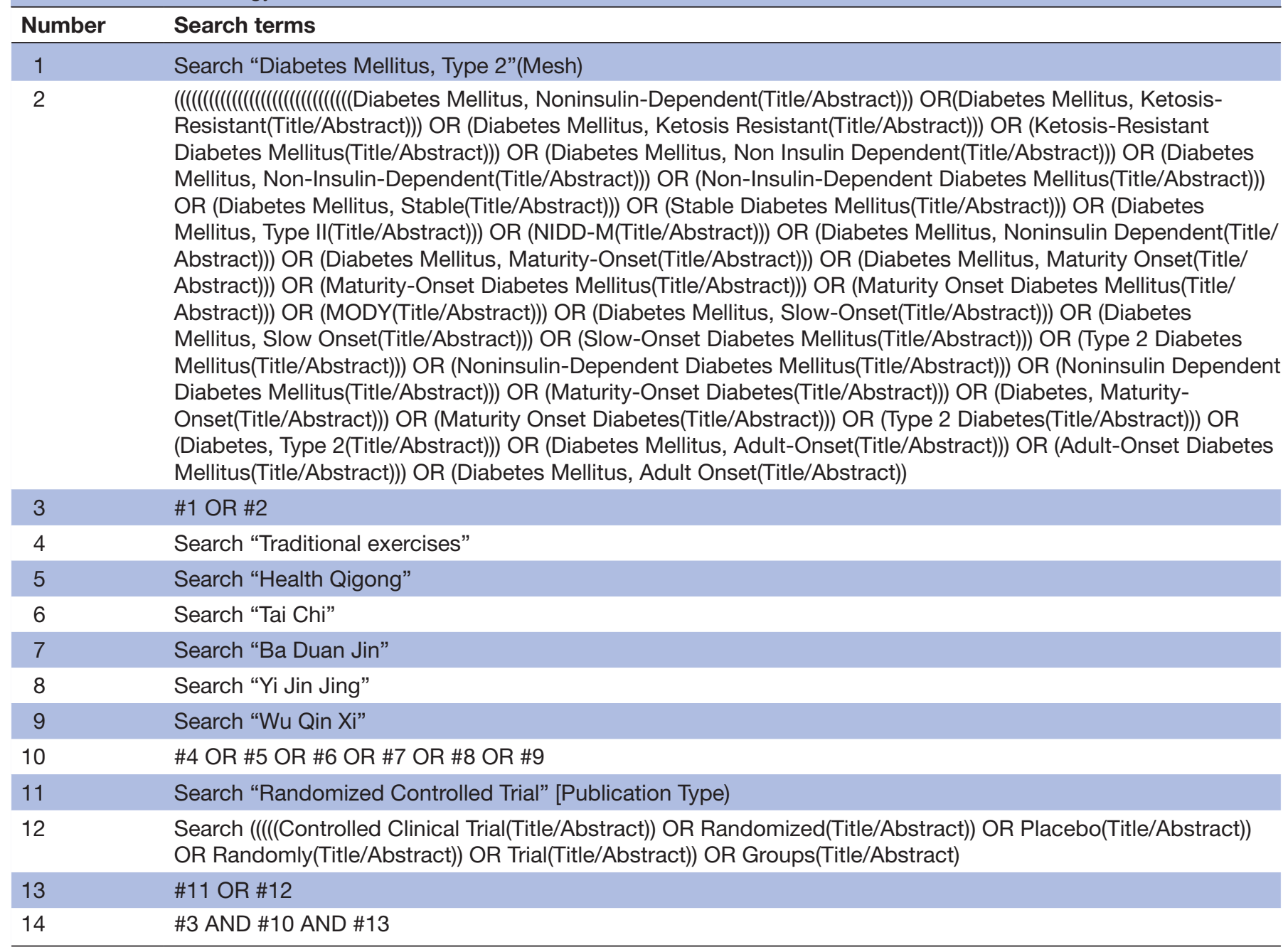

This search strategy was modified as required for other electronic databases. 


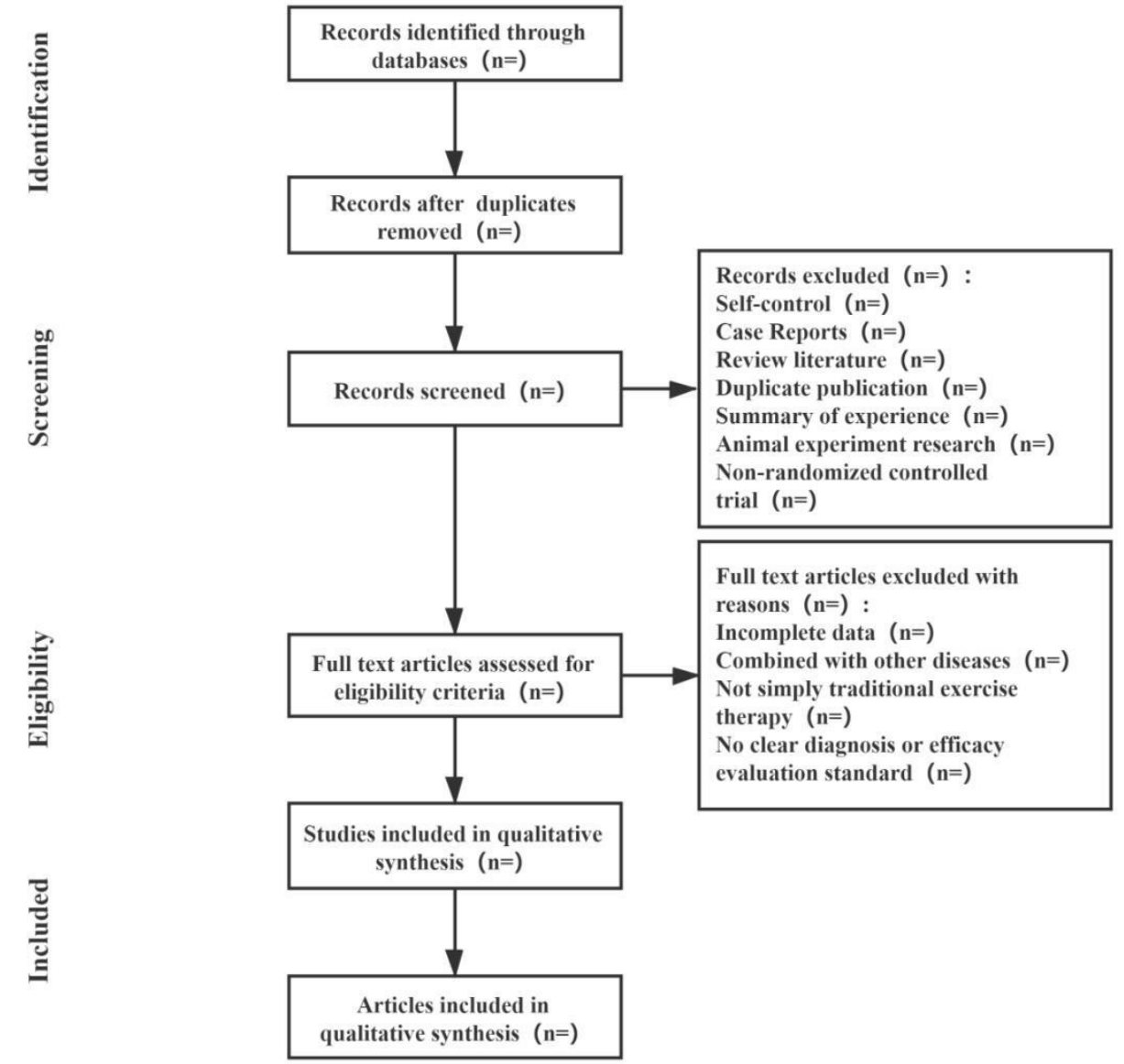

Figure 1 Process of the systematic review.

two researchers conducted preliminary screening independently, extracted data according to a predetermined table, conducted cross-checking and review, recorded the reasons for each excluded study, and invited thirdparty experts to discuss and research different opinions to make the final decision. The Preferred Reporting Items for Systematic Reviews and Meta-Analyses flow chart selected for this process is shown in figure $1 .{ }^{17} 18$

The data extraction content included the basic information of the included literature (including the first author, published journal and year, research topic); the relevant information of the experimental group and the control group in the literature (including the number of cases, disease course, age, intervention measures, treatment course and outcome indicators); the design type and the quality evaluation information of the included literature (eg, random method, blind method, allocation concealment, completeness of outcome data, selective reporting results, other sources of bias).

\section{Study quality evaluation}

According to the quality evaluation standards of the Cochrane System Review Manual, RevMan quality evaluation tools were used to evaluate the methodological quality of the included studies, including random methods, allocation concealment, blinding, completeness of outcome data, selective reporting results and other sources of bias.
Each project was divided into three types of results: high risk, low risk and uncertainty risk. Based on the above descriptions, the two researchers conducted individual induction studies and completed the quality evaluation results of the included literature. If the results were different, third-party experts were invited to help discuss and explain the quality evaluation. Cochrane's standard manual was used for literature quality evaluation and bias risk assessment. ${ }^{19} 20$

\section{Data synthesis and statistical methods}

Pairwise and network meta-analysis

First, the author used RevMan software to analyse the direct comparison results of the literature. Second, for the indirect comparison results, the author used $\mathrm{R}$ and ADDIS software for data merging, statistical analysis and NMA while drawing a network relationship diagram and anecdotal sequence diagram of various intervention measures. ${ }^{21}$ The $\mathrm{R}$ programming language was used to start the netmeta programme and call the Bayesian Markov Chain Monte Carlo (MCMC) algorithm through relevant instructions to realise the network data analysis and mapping of the random effect model data results. ADDIS statistical software uses relevant instructions to call the data results of the random effects model based on the Bayesian MCMC algorithm for prior evaluation and processing. 
A $\mathrm{p}<0.05 \%$ and $95 \%$ CIs were used as the standards of significant difference, and the count data used the OR value as the efficacy analysis statistics; the measurement data used the weighted mean difference (MD) or the standardised MD and indicated that each effect size was expressed with $95 \%$ CI.

\section{Assessment of heterogeneity}

The heterogeneity was assessed by Cochrane analysis. The $\mathrm{I}^{2}$ index was used for statistical heterogeneity assessment, and $\chi^{2}$ was used for subgroup analysis based on heterogeneity factors. The clinical and methodological heterogeneity of the included studies was evaluated, and the levels of fit of the fixed-effect model and the random-effect model were compared. In the absence of significant clinical heterogeneity $\left(p \geq 0.1, I^{2} \leq 50 \%\right)$, a fixed-effects model was used for meta-analysis. If there was significant clinical heterogeneity between the results of each study $(\mathrm{p}<0.1$, $I^{2}>50 \%$ ), the source of the heterogeneity was first analysed, the influence of clinical or methodological heterogeneity was excluded, and the random effects model was used for the meta-analysis. When the data provided by the clinical trial could not be meta-analysed, they were subjected to a descriptive analysis. ${ }^{22}$

\section{Subgroup and sensitivity analyses}

If there is heterogeneity and the data is sufficient, we will try to use subgroup analysis to find out the reasons for the heterogeneity and compare the effects of each group.Data may be compared between patients of different gender, age, course of disease and treatment time.If the result of the meta-analysis was positive and more than three studies were included, R software was used to perform a sensitivity analysis of the statistical results. For each excluded study, the meta-analysis needs was performed again, and the results were compared with the results before exclusion. If there was no substantial change in the comparative analysis, the result was stable. Otherwise, the data results were unstable.

\section{Assessment of inconsistency}

The Node-Split model was used for inconsistency testing. If there was no significant difference between the studies within the subgroup ( $>0.05)$, the heterogeneity of the included studies was small, so the consistency model (consistency model) was used for analysis. Otherwise, the inconsistency model (inconsistency model) was used for analysis. ADDIS software mainly evaluates the final iterative effect of the interchain and intrachain variances through the convergence of the model, that is, the subsequent evaluation through the potential scale reduced factor (PSRF) parameters. The recommended use in this software is to limit the PSRF value, which is more reasonable to be between 1 and 1.05. If the PSRF is not very close to 1 , expansion of the model can continue. Research through software analysis calculations and data analysis found that the PSRF was close or equal to 1, indicating that good convergence performance was achieved, and the results obtained from the consistency model analysis were more reliable. ${ }^{23}$

\section{Publication bias}

According to the recommendations of the Cochrane Handbook, if more than 10 studies were included, RevMan software was used to analyse potential publication bias. If the graph showed inverted funnel-like symmetry, it indicated that the possibility of publication bias was relatively small. If the funnel chart was asymmetric or incomplete, it indicated that there was a greater possibility of publication bias.

\section{DISCUSSION}

Traditional Chinese exercises are aerobic exercises with a strong theoretical basis in Chinese medicine and a long history. Such exercises are a nondrug therapy guided by the theories of Chinese medicine. ${ }^{24}$ At present, an increasing number of studies have confirmed that longterm regular and moderate traditional Chinese exercise has obvious effects on controlling diabetes and lowering blood sugar and can significantly reduce A1C, fasting blood sugar, 2hPG, BMI and other disease indicators to improve the quality of life of patients.

In the case of relatively consistent basic treatment methods, the four traditional exercise therapy adjuvant treatments were clinically comparable. However, at present, traditional Chinese exercise therapy does not have a unified standard or treatment principle for the treatment of type 2 diabetes. Most current studies only report the efficacy of a single traditional exercise and the clinical efficacy and safety of four traditional exercises for the treatment of type 2 diabetes, and comparative studies on NMA are lacking. Therefore, the purpose of this study was to use a high-quality system to evaluate the four commonly used traditional Chinese exercise therapies, to use the NMA method to analyse the blood lipids, blood sugar, BMI and other indicators and to evaluate the quality of life of the four exercise therapies. To determine the effects of four traditional exercise therapies in the treatment of type 2 diabetes, we will sort them according to the pros and cons of the index effects. Then, we will screen out the best evidence of clinical treatment measures and used the analytic hierarchy process to evaluate the quality of the evidence. Our review may provide the best possible exercise therapy options and reliable evidence-based medicine for the clinical treatment of type 2 diabetes, and to a certain extent, can provide some new insights for the auxiliary treatment of type 2 diabetes by exercise therapy.

Contributors $Y J$ and $Y Y$ contributed to the conception of the study. YJ and YY wrote the draft of manuscript, and was revised by $\mathrm{HH}$. The search strategy was developed by all of the authors, YJ and YY will search, extract data, assess the risk of bias and complete the data synthesis. HH will arbitrate in case of disagreement and ensure the absence of errors. All authors approved the publication of the protocol.

Funding This work was supported by the Preliminary Mechanism and Effificacy Evaluation by the excellent scientifific research and innovation teams at Shandong 
University of Traditional Chinese Medicine in the treatment of major diseases (No. 220316).(Corresponding author HH is the project leader).

Competing interests None declared.

Patient and public involvement Patients and/or the public were not involved in the design, or conduct, or reporting, or dissemination plans of this research.

Patient consent for publication Not required.

Provenance and peer review Not commissioned; externally peer reviewed.

Open access This is an open access article distributed in accordance with the Creative Commons Attribution Non Commercial (CC BY-NC 4.0) license, which permits others to distribute, remix, adapt, build upon this work non-commercially, and license their derivative works on different terms, provided the original work is properly cited, appropriate credit is given, any changes made indicated, and the use is non-commercial. See: http://creativecommons.org/licenses/by-nc/4.0/.

\section{ORCID iDs}

Yuqi Jia http://orcid.org/0000-0003-2869-975X

Ying Yu http://orcid.org/0000-0002-3404-0006

\section{REFERENCES}

1 American Diabetes Association. 2. Classification and Diagnosis of Diabetes: Standards of Medical Care in Diabetes-2019. Diabetes Care 2019;42:S13-28.

2 Chinese Diabetes Society. Guidelines for the prevention and control of type 2 diabetes in China (2017 edition). Chinese Journal of Practical Internal Medicine 2018;38:292-344.

3 Xinyue L, Qian L, Guiying S, et al. Research progress on stem cell therapy for type 2 diabetes mellitus and associated complications. Chinese Journal of Comparative Medicine 2020;30:96-102.

4 Qin Z. Nursing common sense to prevent complications of type 2 diabetes. Special health 2020;106.

5 Xingchen Y, Yaling Z, Jingge G. Clinicopathological characteristics and prognosis of minimal change nephropathy with type 2 diabetes mellitus. Chinese General Practice 2021;24:183-9.

6 Balducci S, Sacchetti M, Haxhi J, et al. Physical exercise as therapy for type 2 diabetes mellitus. Diabetes Metab Res Rev 2014;30 Supp 1:13-23.

7 Guanzhou $\mathrm{H}$, Chenji W, Jiang $\mathrm{H}$. The effect of long-term aerobic exercise on the metabolism of blood glucose and lipids in patients with type 2 diabete. Chinese Journal of Physical Medicine and Rehabilitation 2009;31:555-6.

8 Jiaqi Z, Yunchuan W. Exercise therapy and type 2 diabete. World Journal of Integrated Traditional and Western Medicine 2012;7:455-7.
9 Yongjia S, Jiyuan Z, Zejia L. Traditional theory of exercise therapy in the modern value of chronic diseases prevention and control. Clin $\mathrm{J}$ Tradit Chin Med 2015;27:462-4.

10 Fan W, Enfeng S, Yan B. Tai chi for the treatment of type 2 diabetes. Clinical Journal of Traditional Chinese Medicine 2010:205-7.

11 Xilin L, Yingjie W, Dan R. Analysis of the effect of "5123 Baduanjin" on blood glucose metabolism homeostasis in high-risk populations of type 2 diabetes. Asia-Pacific Traditional Medicine 2019;15:124-6.

12 Xinghai L. Research on health Qigong.Wuqinxi on hemorheology of type 2 diabetic patients. Journal of Liaoning Normal University 2007:369-71.

13 Xuejuan M, Linjun Z, Xuezhen F. Analysis of the clinical effect of traditional fitness exercises-Yijinjing on type 2 diabetes. Journal of Frontiers of Medicine 2019;9:65-6.

14 Chinese Diabetes Society. Guidelines for the prevention and control of type 2 diabetes in China (2010 edition). Chinese Journal of Diabetes 2012;20:81-117.

15 Chinese Diabetes Society. Guidelines for the prevention and control of type 2 diabetes in China (2013 edition). Chinese Journal of Diabetes 2014;22:2-42.

16 Zaiying L, Nanshan Z. Internal medicine. Beijing, China: People's Medical Publishing House, 2008.

17 Liberati A, Altman DG, Tetzlaff J, et al. The PRISMA statement for reporting systematic reviews and meta-analyses of studies that evaluate health care interventions: explanation and elaboration. PLoS Med 2009;6:e1000100.

18 Shamseer L, Moher D, Clarke M, et al. Preferred reporting items for systematic review and meta-analysis protocols (PRISMA-P) 2015: elaboration and explanation. BMJ 2015;350:g7647.

19 Hongqiu G, Yang W, Wei L. Application of Cochrane bias risk assessment tool in meta-analysis of randomized controlled study. Chinese Circulation Journal 2014;29:147-8.

20 Jie M, Ying L, Laiping Z. Application and comparison of Jadad scale and Cochrane bias risk assessment tool in quality evaluation of randomized controlled trials. China Journal of Oral and Maxillofacial Surgery 2012;10:417-22.

21 Chao Z, Feng S, Xiantao Z. R software calls JAGS software to realize network meta-analysis. Chinese Journal of Evidence-Based Medicine 2014;14:241-8.

22 Dan W, Junxia ZH, Zhenyun M. Heterogeneity and its treatment in meta analysis. Chinese Journal of Evidence-Based Medicine 2009;9:1115-8.

23 van Valkenhoef G, Tervonen T, Zwinkels T, et al. Addis: a decision support system for evidence-based medicine. Decis Support Syst 2013;55:459-75.

24 Dongmei Y, Xiangdi L, Zhihong L. Research progress of traditional Chinese medicine exercise therapy on diabetes mellitus. Clinical Journal of Chinese Medicine 2017;9:59-61. 